\title{
Conmemorando la Revolución francesa
}

Fue una apoteosis. Entre el 10 y el 17 de julio de 1989, un París renovado - ¿sentido de la grandeza cívica o megalomanía mitterrandianas?- se paralizó para revivir la "Gran Revolución". Con una serie ininterrumpida, auténtica avalancha, de actos culturales, sociales, económicos, festivos, bajo un común denominador político, Francia muestra su capacidad de proyección hacia un mundo en el que, por vez primera en la historia, la democracia liberal resulta ser un valor indiscutido. El día 12 concluyó el Congreso de Historiadores que, presidido por Michel Vovelle, se había iniciado el 6. El 13 dieron comienzo los actos oficiales, con decenas de jefes de Estado y de Gobierno presentes en la ceremonia de los Derechos Humanos en el Trocadero. El mismo día, por la noche, se inaugura la Ópera de la Bastilla: entre las estrellas, Teresa Berganza y Plácido Domingo. El 14, tradicional desfile militar, recepción en el Elíseo, inauguración, en la pirámide del Louvre, de la cumbre de los siete grandes países industrializados: Estados Unidos, Canadá, Japón, República Federal Alemana, Francia, Italia y el Reino Unido. El 15 y el 16, jefes de Estado y de Gobierno se reunieron en el Arco de la Defensa, el gran cubo blanco, con 35 pisos en cada pilar y 300 mil toneladas de materiales, revestido de mármol de Carrara, obra del arquitecto danés Johann Otto von Sereckelsen.

Como culminación la ópera-ballet, La Marsellesa, un impresionante montaje con miles de figurantes, la tercera parte extranjeros -lanzadores de palio italianos, músicos tradicionales franceses, percusionistas africanos, intérpretes ingleses de cornamusa, bailarines de la ópera de Pekín...desfilando la noche del 14 al 15 desde el Arco del Triunfo a la Concordia, ante millón y medio de asistentes. Transmitida por más de cien cadenas de televisión - la mitad en directo- a seiscientos millones de espectadores, el costo del happening excederá los dos mil millones de pesetas, finalizando con el canto del himno por Jessie Norman. El himno por excelencia, del que nos dice Umberto Eco: "Recuerdan Casablanca? Una 
película realizada por americanos con héroes americanos, en una Francia ya rayana en el fondo de su propia vergüenza. Pero cuando la orquesta toca la Marsellesa hasta las prostitutas se echan a llorar. El Yankee Doodle está bien, pero el himno de todas las libertades es la Marsellesa. El autor del espectáculo Jean-Paul Gonde, ilustrador, director artístico de Esquire entre 1970 y 1976, "modelador" de Grace Jones, autor de spots publicitarios formado en el musical americano, nos revela su propósito:

el carácter excepcional de este 14 de julio estará marcado por los ocho mil participantes franceses, norteamericanos, senegaleses, chinos y soviéticos que partirán del Arco del Triunfo para conquistar los 'Champs Elysées'. Durante dos horas, fuera del tiempo, viviremos bajo el sol y la nieve, la bruma y el viento. Oiremos la cornamusa o el tam-tam, la guitarra funk o la gaita. Como en estos sueños que aceleramos para que no terminen, las imágenes más simples encadenarán las más extrañas para mostrar cómo son los hombres en este planeta, dos siglos después de la conmoción de 1789. Esta Marsellesa será, entonces, una poderosa versión contemporánea del himno nacional. Ni desfile ni Carnaval: un espectáculo coreográfico sin antecedentes. Los 'Champs Elysées' deben hacer pensar en los estudios de la época dorada de Hollywood o en las grandes manifestaciones del realismo soviético. En las fiestas tribales de Africa o en los días sudamericanos de auténtico delirio. Y todo transformado para que surja otra cosa: lo nunca visto.

Mas detrás de la fiesta, del espectáculo, un propósito político. Se trataba, en palabras de Jean-Noel Jeanneney, de actuar de acuerdo con la propia Constitución del año III que establecía las "fiestas nacionales para cultivar la fraternidad entre los ciudadanos y ligarlos a la Constitución, a la Patria y a las leyes". En definitiva, que los franceses, gracias a las cadenas de televisión: "se muestren tal como son y enseñen al mundo entero - merced a la imaginación, la emoción, el humor y la magia de Jean-Paul Gonde- el placer de honrar, reunidos, el aspecto luminoso de la Revolución y las virtudes vivientes de su herencia".

Junto a la conmemoración, su crítica, minoritaria pero significativa. Alain Schiffres clamará en Le Nouvel Observateur "iMe han robado mi bicentenario!n, poniendo de relieve un proceso de degradación intelectual que transforma el acontecimiento más importante de la historia de Francia en un spot publicitario. Y las contra-conmemoraciones. La de los integristas de Lefebre -unos siete mil- quienes levantan frente al Museo del Louvre una inmensa guillotina de varios metros de altura y a su lado una cruz. Cerca de veinte mil personas - Liga Comunista Revolucionaria, C.G.T. y Partido Comunista Francés- marcharon desde la plaza de la Bastilla a la Ópera, manifestándose contra la "Cumbre de los ricos, la deuda del Tercer Mundo, el hambre, el apartheid y las últimas colonias". Doscientos mil se reunieron para escuchar en concierto a Renaud y Johnny Clegg. Y se 
denuncian "los shows made in Hollywood" que sustituyen a la fiesta popular, el "contrasentido histórico", y la "falta de gusto".

\section{II}

Para François Furet, uno de los historiadores actuales más influyentes, la revolución francesa, iniciada en realidad por las reformas de Turgot (1774), no concluye sino en 1880. Con Gambetta, con Jules Ferry, la República se consolida. Una nueva legislación seculariza Francia: divorcio, escuela laica, enseñanza obligatoria, libertades de prensa y reunión. La forma republicana de Estado, tan insegura al proclamarse la III República (4-septiembre-1870) —sólo doscientos republicanos frente a cuatrocientos monárquicos son elegidos para la Asamblea en febrero de 1871-, deja de discutirse después de la victoria de aquéllos en las elecciones de 1875 y de la dimisión del presidente, el legitimista Mac Mahon, en 1879.

El 6 de julio de 1880, después de un prolongado debate entre los partidarios del 4 de agosto y los del 14 de julio - toma de la Bastilla, aniversario de la Federación-, la Asamblea consagra esta última fecha como la Fiesta Nacional Francesa ${ }^{1}$. Victor Hugo hará su exaltación, dirigiéndose al Senado: "Esta fiesta es una fiesta popular [...] más que una fiesta popular es una fiesta nacional [...] más que una fiesta nacional, es una fiesta universal [...] señores, el 14 de julio es la fiesta de la humanidad". Su valor histórico y simbólico se mantiene hasta hoy. En 1886, a propuesta de Millerand, la ciudad de París instituye en la Sorbona la enseñanza de la Revolución francesa - convertida en cátedra cinco años más tarde-con Alphonse Aulard, especialista en Leopardi, como encargado de curso primero, titular después. Este Agregé de Lettres, de 37 años, obtuvo el puesto como autor de una publicación sobre los oradores de la Revolución. Cuando abandone su cátedra en 1920, sustituido por Sagnac hará así el resumen de su labor: "He honrado la Revolución, como lo quería el Consejo municipal, es decir, por la verdad. He contribuido por la aplicación del método histórico a introducir la Revolución francesa en la historia. He tenido alumnos en la Sorbona, que han enseñado a su vez [...]. Nadie se atreva ya a escribir sobre la Revolución sin aportar textos y sin citar sus fuentes".

En 1889, el primer centenario de la Revolución tiene lugar en un ambiente de inquietud, producido por el movimiento revisionista del

1 Cfr. R. SAMSON, Les 14 Juillet, fête et conscience national, 1789-1975 (Paris: Flammarion, 1976); M. Ozouf, La fête révolutionnaire 1789-1799 (Paris, 1976). 
general Boulanger. Había que defender la República, es decir, una cultura cuyo principio básico afirma que nadie puede gobernar a otro por razón de su nacimiento, que el poder político radica en el pueblo. Una República que tenía en la Revolución su acta de nacimiento y que suponía el sufragio universal, la igualdad ante la ley, las libertades, la laicidad. Conmemorar era, pues, velar por el legado revolucionario frente a los partidarios de la tradición, de la autoridad monárquica. Unos monárquicos que se movilizaron, con escaso éxito: Albert de Mun promoverá una serie de reuniones que, partiendo de Romans, y de ciudad a ciudad, hasta llegar a París, elaboran una especie de "Cahiers de doléances" de la Contrarrevolución. En Vizille, cerca de Grenoble, Sadi Carnot III, descendiente en línea directa de las glorias del año II y de la vieja guardia del 48, recordaba la reunión de los tres órdenes del Delfinado, que anticiparon allí, en el castillo -21-julio-1788- , la Asamblea Nacional Constituyente, y subrayaba en su discurso la unión política de todos los demócratas ${ }^{2}$. El Aniversario, al que da resonancia la Exposición Universal, conocerá un gran éxito. Su símbolo será la Torre Eiffel, cuya esbelta silueta - 300 metros- proclama el triunfo de la arquitectura de hierro de una Francia orgullosa de ser la patria de los Derechos del Hombre.

Un historiador oficial, Lavisse, difunde a través de sus manuales por todas las escuelas francesas - en las que no faltará tampoco el diccionario de Pierre Larousse- el evangelio laico y republicano, el culto a los héroes de la libertad y de la igualdad. ¿A todos los protagonistas revolucionarios por igual? No, ciertamente. En su discurso inaugural de cátedra, Aulard invoca a Quinet, quien no se dejó cegar por "la masa enorme e inmóvil de la Revolución francesan. No hay que confundir a Mirabeau con Danton, a Robespierre con los girondinos. $\mathrm{Y}$ es que:

el gran descubrimiento de los años jubilares —nos dice Mona Ozouf- es que el objeto conmemorado no es homogéneo, que la Revolución francesa no es una evidencia, sino un problema [...]. La paradoja de estas festividadades tendidas a lo homogéneo es, en primer lugar, que deben renunciar al relato ligado; éste pondría inevitablemente en evidencia el tiempo entrecortado del decenio revolucionario, sus rupturas dramáticas, mientras que la conmemoración quiere meter por todas partes una trabazón eufórica ${ }^{3}$.

Junto a lo conmemorable hace su aparición lo inconmemorable. Lo que no debe recordarse es la guillotina, el Terror, Robespierre. $\mathrm{Y}$ aun sobre

2 Cfr. P. METO, Le centenaire de la révolution dauphinoise. Vizille, un mythe républicaine (Grenoble: Presses Universitaires de Grenoble, 1988).

3 M. Ozouf, "¿Se puede conmemorar la Revolución Francesa?», Debats, 25 (septiembre, 1988), p. 7 
Danton, que vive entonces su hora de gloria historiográfica, sobre su biografía, se pasa como sobre ascuas: cuando en 1888 se descubre oficialmente su estatua, erigida en su lugar de nacimiento, Arcis-sur-Aube, "por una debilidad en la voz (el orador, Mr. Robinet) ha tenido que restringir considerablemente - nos informa la prensa- la exposición biográfica que se proponía desarrollar". Es lícito pensar, observa Ozouf, que "tal discreción se debe no a la laringe de Robinet, sino a la incomodidad del relato. La misma celebración de Condorcet superó con éxito la proeza de no soltar ni una sola palabra sobre el Terror ${ }^{4}$. Unos años después, sin embargo, con ocasión del estreno de Thermidor, de Sardou - enero1891-, pieza justificativa de este momento histórico, ante las manifestaciones tumultuarias de los nostálgicos del jacobinismo, que obligan a cerrar el teatro, Clemenceau pronunciará en la Asamblea su frase célebre: "Señores, lo queramos o no, nos guste o nos moleste, la Revolución francesa es un bloque del que no se puede cambiar nada porque la verdad histórica no lo permite".

Retengamos del primer Centenario dos problemas hoy todavía vivos, aun cuando no se planteen ya de idéntica manera. El enfrentamiento derecha-izquierda, y la legitimidad de fragmentar la Revolución: ¿tenemos lógica, necesariamente, que tomarla globalmente, o podemos distinguir y valorar fases y protagonistas?

Cincuenta años después, en 1939, las circunstancias son muy diferentes. La libertad retrocede ante los totalitarismos en el poder. La celebración de los 150 años de la Revolución está ensombrecida por la amenaza alemana. Se exalta la unidad de Francia, se sitúa el acontecimiento en una perspectiva internacional. El 14 de julio, bajo la lluvia, las tropas desfilan ante Daladier, quien invoca el espíritu del 89, "nueva religión", "movimiento de amor", y explica el papel de aquéllas: "Este ejército que habéis aclamado es el guardián de nuestras libertades y tiene la potencia necesaria para responder a todos los ataques que pongan en peligro a nuestro país". Los actos, con pocos medios, son un semifracaso. Eco de la coyuntura, estaba prevista una gran ceremonia el 20 de septiembre en Valmy, celebrando la unión del ejército con la República, en la línea postulada por Jaurés, en El nuevo ejército. Mas las fuerzas militares estaban ya ocupando sus posiciones de combate.

En este momento de exaltación de la unidad de Francia, falta por completo la unanimidad en torno a la conmemoración de la Revolución. La tradición monárquica por un lado tiene, a despecho de su escasa fuerza política, una influencia indudable en los medios intelectuales, a través de

4 Ibid., p. 8 
la "Acción Francesa" de Charles Maurras, cuya Encuesta sobre la Monarquía se empezó a publicar en el verano de 1900, en la Gazette de France. Se reedita por Marabout La Revolución Francesa de Pierre Gaxotte, que había conocido un gran éxito en el momento de su publicación (1928). En 1937 aparecen, recogidas y ordenadas por Pierre Chardon, autorizadas por el propio maestro, Mes ideés politiques de Maurras. "Acción Francesa", junto a los nombres citados, Madélin, Cochin, Bainville, Massis..., "suministran fundamentos razonados a la nostalgia y proporcionan un arsenal ideológico a los enemigos de la Revolución francesa" 5 . La monarquía "tradicional, hereditaria, antiparlamentaria y descentralizada", ámbito natural de expresión del nacionalismo, aparece como "el reverso de una República" heredera de la Revolución (P. Nora). La Revolución francesa, que rompió la continuidad histórica de Francia e instauró el principio igualitario, fue una inmensa catástrofe para el país. Je suis partout dedica su número 30, de junio de 1939, "a aquellos que han luchado, con todos los medios a su alcance, contra la barbarie revolucionaria, y, particularmente, a los campesinos de la Vendée, que murieron por la verdad, así como a la alta y sagrada memoria de Charlotte de Corday".

La tradición liberal se vincula a 1789, al concepto de una Revolución conclusa en sus principios fundamentales. Herriot, en su discurso del 5 de mayo de 1939 en la Galería de los Espejos, lo manifiesta sin ambages: "Por mi parte, no acepto que se diga que la Revolución es un bloque. Los odios mutuos de los hombres han obstaculizado el movimiento de las ideas. Robespierre consultó por demasiado tiempo el espectro de Rousseau".

Mientras tanto, la interpretación marxista de la Revolución empieza a ser hegemónica: el socialismo continúa la obra revolucionaria. En mayo de 1889, Partido obrero decía: "la Revolución es permanente. Es de eterna actualidad [...]. Porque las causas que la producen están, por siglos todavía, en el corazón del hombre». Continuidad y también futuro. Jean Jaurés publica su Historia socialista de la Revolución Francesa (1903), abriéndola así a un porvenir que será su culminación. Y en octubre de 1917, Revolución francesa y Revolución rusa se funden en una misma matriz ideológica. Un hombre se eleva ahora, Robespierre. Un período también, el Terror, legitimado desde la dictadura del proletariado. En 1920, Mathiez, un historiador universitario, publica su folleto El bolchevismo y el jacobinismo. Allí escribe: "Jacobinismo y bolchevismo son, con igual título, dos dictaduras de clase, que operan con los mismos medios: el terror, la requisa y los impuestos, y se proponen un fin semejante, la transformación

5 F. SIRINELlI, "Les greffe maurrasienne", L'Histoire, 113 (1988), p. 87. 
de la sociedad y no sólo de la sociedad rusa o de la sociedad francesa, sino de la sociedad universal".

En el espíritu del Frente Popular se encarga a Renoir un film, La Marsellesa, notable evocación del 10 de agosto de 1792. Los comunistas celebrarán aparte la Revolución, culminada en la gran concentración del estadio Buffalo. Una Revolución que no ha concluido -nunca concluye, para la extrema izquierda-. Maurice Thorez dirá el 25 de junio de 1939: "La clase obrera no tiene que acabar 1789. Lo que ha de hacer es cumplir un 1939, un 1940, un 1945 ó 1946, que sea el 1917 del Pueblo de Francia..

Para celebrar 1989, se creó la "Mission de conmémoration du Bicentenaire de la Révolution Françcaise et de la Déclaration des droits de l'homme et du citoyen", presidida finalmente, después del fallecimiento de sus antecesores en el puesto Michel Baroin y Edgard Faure, por un profesor de Historia Contemporánea, Jean-Nöel Jeanneney. La misión, con un presupuesto total de 129 millones de francos -en Australia se destinaron 290 a realzar su bicentenario-, asistida de un comité interministerial, dependió directamente del primer ministro, teniendo como función apoyar y coordinar las iniciativas particulares dando sentido al conjunto total de actuaciones, y realizando por su parte algunos proyectos específicos.

Hasta llegar a la última semana - ya descrita al principio- los actos se multiplicaron, yendo desde lo banal a lo importante. En los Jardines de las Tullerías se celebra un "cross revolucionario", en el que los corredores visten los colores rojo, blanco y azul, y en el "Pré catelan" tiene lugar la cena del bicentenario: preparado por Joel Robuchon se sirve el "Capón a la sal gorda", plato típico del París de la época, según Claude Quétel, autor de La Bastille y director de investigaciones del CNRS ${ }^{6}$. En TV varios miles de franceses, en un proceso escenificado, absuelven a Luis XVI; Nina Companez presenta La grande cabriole, un serial novelado sobre la Revolución, y en octubre en "Antenne 2" se da Le souffle de la liberté, un gran documental histórico en cuatro partes, de Daniel Costelle, sobre los avatares a lo largo de los dos últimos siglos de los derechos del hombre. En 1983, por encargo gubernamental, Andrzej Wajda realizó una espléndida película, Danton, con Gerard Depardieu en el papel del tribuno francés, y Wojciech Pszoniak en el de Robespierre: el filme, al centrarse en el análisis del Terror, desagradó a las autoridades. Un cortometraje en relieve de diez minutos producido por Ex Móchina, una filial de Thompson Digital Images, y realizado mediante ordenador, revive el París de 1789. Robert Hosseis, después de L'Affaire du Courrier de Lyon, presenta en el Palais

6 Cfr. C. QUÉTEL, La Bastille. Histoire vrai d'une prision légendaire (Paris: Robert Laffont, 1988). 
des Congrés La Liberté ou la Mort: es la versión "Kitsch" de la Revolución. El Thêatre Job de Burdeos ofrece 1789, una nueva versión del texto puesto en escena por Ariane Mnouchkine en 1969, primero en el Piccolo de Milán, después en la Cartoucherie de Vincennes. Estalla el gran acontecimiento musical y coreográfico de la celebración, $1789 \ldots$ y nosotros, de Maurice Bejart, ingenuamente ecologista y reivindicativo de Robespierre ("entendió - según el programa de mano- el fondo esencial de lo que debiera ser la República"), pero que es una fiesta. En su estela, el Ballet Teatro de L'Arche de Maguy Marín nos brinda, con propósito desmitificador, "Et qu'est ce que ça m'a fait à moi".

Se reedita la vieja novela de Robert Margerit, e Yves Amiot publica Les diamants de l'Higonne. $\mathrm{Y}$, literatura al fin y al cabo, una nueva edición de los Portraits de la Révolution et l'Empire, de Charles Nodier. Los documentos revolucionarios se cotizan alto en las subasta de Druot: 600.000 pesetas por una carta de Danton; 70.000 más por una de Robespierre, y sólo 200.000 por una lista de 75 personas, esperando su última hora en la Conciergerie, firmada por Fouquier-Tinville. Muchas exposiciones: La Revolución y Europa, en el Grand Palais (1.140 obras, procedentes de 15 países, pinturas, esculturas, estampas, objetos de todo tipo, de domésticos a científicos, seleccionados por un comité de expertos); David, en el Louvre; Los arquitectos de la Revolución, en la Cité des Sciences et de l'Industrie... Entre más de 500 libros publicados, algunos fundamentales: La Revolution, 1770-1880, de François Furet (Hachette); el Dictionnaire critique de la Révolution Française, dirigido por F. Furet y Mona Ozouf (Flammarion); L'État de la France pendan la Révolution, a cargo de Michel Vovelle (La Découverte); el Atlas de la Révolution Française (Ecole des Hautes Etudes en Sciences Sociales); la Histoire provinciale de la Revolution Française, coordinada por Louis Bergeron y Jean-Luc Mayaud (Bibliothéque Historique Privat); L'Invention de l'bomme moderne, de Robert Muchembled (Fayard); Les républicains des lettres, gens de culture et Lumieres au XVIIIe siècle, por Daniel Roche (Fayard); Aux armes et aux arts citoyens, les arts et la Révolution 1789-1799, dirigido por Philippe Bordés y Régis Michel (Adam Biro), etc. En fin, florecen los coloquios internacionales: Chicago (septiembre, 1986), Oxford (septiembre, 1987): La Revolución francesa y la cultura política moderna (1789-1848), promovido por la Escuela de Altos Estudios en Ciencias Sociales, y entre el $6 \mathrm{y}$ el 12 de julio en La Sorbona, el que bajo el título La Revolución y su imagen, organizó Vovelle. 
III

Mas, ¿cuál ha sido el sentido de la política oficial ante el Bicentenario? Ante todo un propósito cívico, la exaltación de los ideales de la Revolución, en lo que tienen de llamamiento a todos los hombres:

En 1889 - dice Jeanneney - se trataba de afirmar la República, haciéndola segura de sí misma y de su destino. Hoy día, el compromiso es diferente, la República está menos amenazada. Pero una cuestión se nos plantea y orienta nuestro trabajo: ¿Cómo podría esta conmemoración contribuir a que los ideales de la Revolución se encarnen mejor, se atente menos contra ellos, se les burle en menor grado, en Francia y, sobre todo, en el resto del mundo? Su mensaje, en suma, continúa siendo tan subversivo como lo fue siempre ${ }^{7}$.

Se trata, pues, de poner de relieve la herencia moral de la Revolución: los Derechos del Hombre y de la ciudadanía, el fin de los privilegios y de las exclusiones, de la religiosa especialmente, la instauración de una administración eficaz, el sufragio universal y las leyes sobre la enseñanza ${ }^{8}$. En este sentido, aun cuando se habla, como lo hizo Clemenceau, de considerar la Revolución "en bloque", 1789 aparece en el centro del Bicentenario. A este año corresponden, en efecto, casi todas las grandes fechas que centraron los principales momentos de la celebración: 5 de mayo ("marcha" de los Estados Generales hacia Versalles, con más de medio millón de personas, muchas disfrazadas); 17 al 20 de junio (jornadas de "Fraternidad" en conmemoración del juramento del Jeu de Paume, que culminarán con la inauguración de un monumento conmemorativo de los Derechos del Hombre); 10 al 17 de julio (momento central con discurso del presidente de la República, coincidiendo con la cumbre en París de los siete grandes países industrializados de Occidente, lo que acentúa la dimensión internacional del Bicentenario); 4 de agosto (fin de los privilegios); 26 de agosto (Declaración de los Derechos del Hombre y del Ciudadano); y la excepción, 20 de septiembre (aniversario de la batalla de Valmy y del nacimiento de la República en 1792), con la presencia del jefe del Estado.

En resumen, según expresión de Agulhon: "Nada de catecismos, nada de discursos oficiales sobre cada punto de la historia. Un bicentenario que se limite a recordar 1789, y a honrarlo como punto de partida o (si se prefiere) etapa fundamental de la modernidad liberal, y

7 Cfr. J.-N. JEAnNENEY, entrevista en Le Nouvel Observateur, 15-21 julio, 1986.

8 Cfr. "Liberté, egalité, fraternité... des valeurs subversives", entrevista con J.-N. JEANNENEY, L'Histoire, 113, pp. 122-123. 
como tiempo de proclamación de los Derechos del Hombre y del Ciudadano" ${ }^{9}$.

Hay, por tanto, un propósito definido de que la conmemoración sirva para unir al país, de superar las viejas divisiones. Así, como dijo Edgard Faure, afirmando la carencia de sentido de las polémicas del tipo "era o no preciso hacer la Revolución",

nos es necesario extraer dos lecciones. Una de ellas, es la de una gran reconciliación francesa. Es preciso que los descendientes de los 'Vendéens' y los de los combatientes de Valmy comprendan que sus antepasados eran hombres dispuestos al sacrificio por el interés nacional, por el interés de los hombres. Es preciso llegar a esa soldadura, a esa reconciliación en el sentido casi eclesial del término, y a la idea de terminar con la oposición derecha e izquierda, que transforma Francia en dos bloques que parecen impermeables.

Hay, en fin, que abandonar "la lógica paranoica que quiere que haya dos campos enfrentados" (Gauchet). Una segunda finalidad ha sido la didáctica. Las encuestas eran desoladoras: un sondeo de Le Figaro muestra que el 40 por 100 de los franceses ignora que Luis XVI murió guillotinado. Otro de "Antenne 2" que asimismo otro 40 por 100 cree que el general De Gaulle jugó un papel importante durante la Revolución francesa. El Bicentenario se dirige a la juventud - la edad media de los diputados en la Asamblea Legislativa excedía escasamente los veinticinco años-: "Pienso que [...] puede ser ocasión para que los niños y los adolescentes conozcan precisamente los acontecimientos revolucionarios, dominio que la enseñanza ha despreciado. La mayor parte de los jóvenes no conocen la diferencia entre girondinos y montagnards" ${ }^{10}$.

La propuesta gubernamental incide sobre una Francia en la que la democracia liberal consolidada, con un prestigio reforzado por los acontecimientos recientes en los países del Este y en China, limita la radicalidad del enfrentamiento derecha-izquierda. Mas la Revolución francesa sigue siendo objeto de un fuerte debate. ¿Por qué? Para Michel Winock los imperativos económicos, las interdependencias exteriores, las

9 M. AgulHon, "¿Hay que tener miedo de 1989?», Débats, 25 (septiembre 1988), pp. 31-32.

10 J.-N. JEANNENEY, op. cit. Las publicaciones dirigidas a los niños abundan. Véanse, entre otros libros, A Paris sous la Révolution (Casterman), Au coeur de la Révolution (Albin Michel); La Révolution française. Le journal d'Antoine Vestbois (27 Avril 178927 Juillet 1794) (Centurion-Okapi); Les soldats de la Révolution française, de Lilian y Fred FUNKEN (Castermen); Alain Decaux raconte la Révolution française aux enfants (Perrin); La Révolution française au jour le jour, par Denys PRACHE; La Révolution française. Elle inventa nos rêves, de Bernard EPIN (Messider)... 
obligadas solidaridades reducen el campo de elecciones posibles entre derecha e izquierda:

En este contexto, el pasado — cristalizado, endurecido- mantiene sus encantos; recurrir a él refuerza las diferencias con más seguridad que las elecciones presupuestarias, estratégicas o diplomáticas [...]. Quizás la llamada seductora de la Revolución no es más que una transferencia de las mediocres rivalidades contemporáneas a la edad de oro de las guerras políticas para darles un poco de mordiente ${ }^{11}$.

El ruido de las diferencias, aparentemente muy vivas, recubre así un fondo no excesivamente conflictivo. Incluso la controversia se civiliza en sus formas ${ }^{12}$.

Las posiciones siguen siendo las tradicionales. Utilizando la terminología clásica, heredada de la revolución, a la derecha se sitúa Pierre Chaunu, quien confiesa sentir vergüenza de una Revolución que declaró la guerra a Europa, privando a los pueblos de su libertad:

No es posible conmemorar 1789, olvidando las desgracias posteriores de la Revolución, la guerra, el genocidio vendeano y la ruina duradera de un país que era próspero. El balance es absolutamente negativo. De un lado, la abstracción de los derechos del hombre; del otro, matanzas muy reales y un desastre económico. Para mí, la elección es clara y, si yo no tengo los medios de rehusar la conmemoración - aún menos de impedirla-, debo al menos decir que se trata de una superchería con la que se nos viene ensordeciendo desde hace ocho años ${ }^{13}$.

En una posición semejante, Claude Levi-Strauss:

La Revolución ha puesto en circulación ideas y valores que han fascinado a Europa, después al mundo, y que proporcionaron a Francia, durante más de un siglo, un prestigio y un fulgor excepcional. Cabe preguntarse, sin embargo, si las catástrofes que se han abatido sobre Occidente no tienen en ella su origen [...] se ha hecho pensar a la gente que la sociedad se funda en el pensamiento abstracto, cuando en realidad está hecha de costumbres, de usos, y que, tritu-

11 M. WINOCK, "La gauche, la droite et la Révolution", L'Histoire, 113, p. 100

12 En 1988 se reeditó Sur la Révolution française. Contribution à l'bistoire de la révolution bourgeoise (1965-1966), de Claude MOZAURIC, un clásico del momento de la polémica abierta por el libro de FURET y RICHET, La Révolution française (1965). En la p. 61, MAZAURIN lamenta haber empleado en la primera edición la frase "parti pris antinational".

13 Entrevista con P. CHAUnu, Le Monde de la Révolution Française, n. ${ }^{\circ} 4$, mensual, abril 1989, p. 26. De este autor, Le Grand Déclassement. A propos d'une conmémoration. Robert Laffont (1989). 
rando éstos bajo las muelas de la razón, se pulverizan géneros de vida fundados en una larga tradición, se reducen los individuos al estado de átomos intercambiables y anónimos. La libertad verdadera no puede tener más que un contenido concreto ${ }^{14}$.

Jean-Marie Benoist rechaza la conmemoración "en bloque" de la Revolución. Hay que trazar una infranqueable línea de demarcación entre el humanismo, fundado en las luces, de la Declaración de 1789, y la desviación sangrienta que se inicia en 1791 y culmina en 1792 y 1793 :

Conviene que la Francia de 1989 considere de forma crítica los ejemplos desastrosos que nuestro país ha dado al mundo a partir del período 1792-1794: prototipos y arquetipos de todo terror revolucionario y de todo despotismo moderno [...]. Querer matar a todos los miembros de una categoría sin excepción, entonces los aristócratas, después los judíos, es la forma recurrente de genocidio, con su cortejo de delaciones. La ley de sospechosos establecida por el Terror prefigura el tipo de persecución retomado por la Tchéke bajo Lenin y prolongado por Stalin y el Goulag. La masacre de vendeanos: Matad a todos, azules y blancos, hombres, mujeres y niños, es la prefiguración de la Camboya de Pol Pot [...]. La Revolución francesa, por la generalización de la guillotina, inventa las bodas del maquinismo y el asesinato político, como mostró Lauren Dispot en un libro de los años $1970^{15}$.

La izquierda con Vovelle -director del Instituto de Historia de la Revolución Francesa de París, presidente de la Comisión de Investigación histórica para el bicentenario de la Revolución Francesa- como cabeza de fila, mantiene la tradición marxista: la Revolución francesa como revolución social; defensa, ahora crecientemente matizada, del jacobinismo y del Terror revolucionario:

creo que la utilización de este término ['genocidio' para referirse a la guerra de la Vendée] no es apropiada. Fue una guerra terrible, cruel, a la antigua, pero sin intención exterminadora y sin carácter racial. Este énfasis sobre la violencia ha conducido a una especie de exorcismo sobre la Revolución, que puede ocultar sus aspectos positivos, y que hace el diálogo imposible porque intervienen elementos pasionales ${ }^{16}$.

14 Cfr. C. LÉvi-STRAuss, De près et de loin, entrevistas con Didier Eribon, Ed. Odile Jacob (Paris, 1988).

15 J.-M. BenOIST, "Au nom des Lumières...", Le Monde, viernes, 6 de junio 1989.

16 M. Vovelle, "Je refuse d'être le Dukakis de François Furet". Propuestas de Antoine DE BAECQUe y de Olivier SALVATORI, Le Monde de la Révolution Française, núm. 2, pp. $28-29$ 
En el Terror, según Max Gallo, hay que ver a la vez «un proceso de barbarie y el mecanismo por el cual una gran parte del pueblo marchó a las fronteras a defender Francia, es decir, la libertad y los derechos del hombre. Comprender la Revolución es comprender, a la vez, ambas cosas" ${ }^{17}$. En fin, no faltan tampoco muestras del viejo estilo. Para Anne Soprani, Olympe de Gouges "está a la derecha, lo que modifica la imagen de su combate feminista" ${ }^{18}$; y Annette Rosa elogia, desde el simplismo "gauchiste", al sanguinario Marat en estos términos: "Uno de los hombres políticos más clarividentes de su tiempo, el primer teórico de la democracia completa y de la violencia revolucionaria" "

$\mathrm{Y}$ en el centro, finalmente, y es la posición que en definitiva se ha impuesto, François Furet. La Revolución, según este historiador, ha concluido al crearse el adecuado, es decir representativo, sistema de la democracia parlamentaria. Tuvo un primer final, con la Tercera República y la síntesis realizada por Ferry y Gambetta, y un segundo hará unos diez años: rápido declive del Partido Comunista, abandono de la idea nacional - ¿cómo hacer comprender a un joven de hoy lo que fueron las campañas napoleónicas o incluso la guerra del 14?»; conclusión del gaullismo y de cierta cultura de la nación; consenso sobre una monarquía democrática —la elección de un presidente cada siete años e incluso reelegirlo no se había visto desde hace doscientos años —; consejo constitucional - "la idea de una magistratura a cargo de la constitucionalidad de las leyesn-, y término de la guerra religiosa —los franceses han acabado por reconocer, al finalizar el siglo $\mathrm{xx}$, que la religión es un asunto privado"- Asistimos al término de la "revolución a la francesa", es decir a la creencia colectiva en la "gran noche". $\mathrm{Y}$ es harto dudoso su futuro en las sociedades democráticas. La izquierda abandona la convicción de que la Revolución democrática debe ser seguida necesariamente por otra revolución socialista o comunista.

En las postrimerías del siglo xx se produce justamente lo contrario: la idea democrática se ha convertido en el porvenir de la idea socialista, e incluso en el futuro

17 "La liberté ou la Terreur", Le Nouvel Observateur, 4-10 mayo, 1989, p. 13. Cfr. Max GALLO, Lettre ouverte à Maximilien Robespierre sur le bicentenaire de la Révolution française (Albin Michel, 1989).

18 Cfr. A. Soprani, La Révolution et les femmes. 1789-1796 (M. A. Editions, 1988).

19 Cfr. A. RosA, Citoyennes. Les femmes et la Révolution française (Messider, 1988). Véase, para situar correctamente la figura de Marat, el artículo de Mona Ozouf, en el Dictionnaire critique de la Révolution, dirigido por ella misma, junto con FURET, capítulo "Acteurs". 
mundo comunista. Contemplad lo que nos llega desde la URSS: el mercado, los derechos del hombre, la democracia, denunciada como "formal" durante largo tiempo. En este sentido, 1789 ¡es más actual que nunca! ${ }^{20}$.

\section{ANTONIO MORALES MOYA \\ Universidad de Salamanca}

La conmemoración del segundo centenario de 1789 en París, culminada en la semana del 10 al 17 de julio, constituyó un espectáculo masivo a los ojos del mundo que, por los medios empleados (TV, ópera, filmografía, etc.), sirvió espléndidamente a los fines a que estaba destinada: mostrar al resto del mundo la inquebrantable identidad democrática de la República francesa en el mundo actual. Aparte de algunos críticos exquisitos, el acontecimiento en general se vio con gran unanimidad como una imagen homogénea. Durante el siglo pasado (1889) y en 1939, sin embargo, hubo una oposición más fuerte, formada por políticos monárquicos o contrarrevolucionarios. Lo curioso es que esta homogeneidad de 1989 fuera compatible con una gran ignorancia de la historia real por parte de la juventud francesa: a ellos - y al gran público del resto del mundoes a quienes se dirigieron esos espectáculos, dotados a veces por ello de gran sentido pedagógico.

The Bicentennial of the French Revolution in Paris, in the week of July 10 through 17, 1989 was such a massive, elaborate event that, given the media employed (TV, opera, films, etc.), attained brilliantly the objective which from the outset its organizers intended: to show the rest of the planet the unbreakable democratic identity of the French republic in the world today. Leaving a few uncompromising critics aside, the event was perceived generally as presented coherently. However, on the occasion of the First Centennial, in 1889, as well as the 150th aniversary, in 1939, criticism was harsher, voiced by monarchists and unabashed opponents of the Revolution. Significantly, the consensus of 1989 coincided with widespread ignorance of French history among the country's young; and it was at them, as well as the gneral public in the rest of the world, that the event was directed, with a powerful educational thrust.

${ }^{20}$ F. FURET, "L'Idée démocratique et l'avenir de l'idée socialiste". Propuestas de Antoine DE BAECQUE y de Olivier SALVATORI, Le Monde de la Révolution Française, n. ${ }^{\circ}$ 2, mensual, enero 1989, p. 28. "Histoire de l'idée révolutionnaire", una entrevista con François FURET, Magazine Litteraire, 258 (octubre 1988), pp. 18-20. 